\title{
EFFECT OF CROSS-SECTIONAL GEOMETRY ON THE RPA PLASMONS OF QUANTUM WIRES
}

\author{
C.R. Bennett ${ }^{\dagger}$, B. Tanatar ${ }^{*}$, N.C. Constantinou ${ }^{\dagger}$ and M. Babiker ${ }^{\dagger}$ \\ tDepartment of Physics, University of Essex, Colchester C04 3SQ, England \\ *Department of Physics, Bilkent University, Bilkent 06533, Ankara, Turkey
}

(Received 15 September 1994 by R.T.Phillips)

\begin{abstract}
The effect of cross-sectional geometry on both the intrasubband plasmon and intersubband plasmon of a quantum wire is investigated within a two-subband RPA scheme. Exact analytical electronic wavefunctions for circular, elliptical and rectangular wires are employed within the infinite barrier approximation. It is found that for fixed cross-sectional area and linear electron concentration, the intrasubband plasmon energy is only marginally dependent on the wire geometry whereas the intersubband plasmon energy may change considerably due to its dependence on the electronic subband energy difference.
\end{abstract}

Keywords: A. nanostructures, A. semiconductors, D. dielectric response.

\section{INTRODUCTION.}

There has been considerable interest in the plasmon spectrum of quasi-one dimensional (Q1D) semiconductor systems, both theoretical [1-7] and experimental [8-10]. The experimental confirmation of the Q1D long wavelength intrasubband plasmon via inelastic light scattering by Goni et al [8], strongly suggests that such systems behave as Fermi liquids, in agreement with the theoretical conclusions of $\mathrm{Hu}$ and Das Sarma [11] .

Quantum wire cross-sections vary depending on the fabrication process employed. In particular, circular [12], so-called ' $\checkmark$-groove' [13], and 'arrowhead' [14] devices have recently been fabricated. The cross-sectional geometry may therefore be quite different, and as such, it is important to investigate any effects that different cross-sections have on the plasmon energy. The possible effects of different wire cross-sections has recently been raised by Anthony and Kelly [15] in the context of quantum wire lasers.

In this communication we calculate the plasmon dispersion for circular, rectangular and elliptical crosssections within the infinite barrier approximation. These three geometries are chosen for mathematical convenience since the effective mass Schrodinger equation is separable, with exact analytical solutions. Any differences between them will be indicative of differences between more realistic cross-sectional geometries [15] which inevitably require the numerical solution of the effective mass equation. We defer consideration of the plasmon spectra for quantum wires with more complex cross-sections to a future detailed article.

\section{THEORY AND NUMERICAL RESULTS.}

The model we employ is a two-subband model at zero temperature and we assume that only the first subband is occupied. In all calculations the linear electron density, $n$, is taken to be $5 \times 10^{5} \mathrm{~cm}^{-1}$ which corresponds to a Fermi wavevector $k_{f}$ of $7.85 \times 10^{5} \mathrm{~cm}^{-1}$ and a Fermi energy, $E_{f}$ of $3.5 \mathrm{meV}$. The material parameters are those appropriate for GaAs. The quantum wire has an effectively infinite length $L$ (in the $z$-direction) and the electrons are assumed to be confined by an infinite potential. We consider wires of circular, elliptical and rectangular cross-sections. In the circular case the normalised wavefunctions transverse to the wire axis are Bessel functions of integer order multiplied by a simple angular exponential [2,7], for elliptical wires the transverse wavefunctions are Mathieu functions $[16,17]$, and for the rectangular wire they have the usual sinusoidal form. For brevity we do not dwell on the properties of these wavefunctions, but just mention here that for wires of equal area, the resultant energy separation between the ground state and the first excited state, $\Delta E$, is different for the three geometries. This has a noticeable effect on the depolarisation shift which has a well documented dependence on this splitting [e.g.3].

The dielectric matrix is obtainable within the randomphase approximation (RPA) and is given by $[2,3]$

$$
\varepsilon_{i j m n}(q, \omega)=\delta_{i m} \delta_{j n}-V_{i j m n}(q) \Pi_{i j}(q, \omega)
$$

where the indices label the electronic subbands. In the above $\Pi_{i j}(q, \omega)$ is the generalised irreducible polarization

$$
\Pi_{i j}(q, \omega)=2 \sum_{k} \frac{f\left(E_{i, k+q}\right)-f\left(E_{j, k}\right)}{\hbar \omega+E_{i, k+q}-E_{j, k}+i \eta}
$$

and

$$
V_{i j m n}(q)=\frac{e^{2}}{2 \pi \varepsilon \varepsilon_{o} L} F_{i j m n}(q)
$$

is the Coulomb matrix element with the structure factor given by

$$
\begin{gathered}
F_{i j m n}(q)=\int d^{2} r_{1} \int d^{2} r_{2} \psi_{i}^{*}\left(\mathbf{r}_{1}\right) \psi_{j}\left(\mathbf{r}_{1}\right) \\
\times \mathbf{K}_{0}\left(q\left|\mathbf{r}_{1}-\mathbf{r}_{2}\right|\right) \psi_{m}^{*}\left(\mathbf{r}_{2}\right) \psi_{n}\left(\mathbf{r}_{2}\right)
\end{gathered}
$$

which is non-zero if $i+j+m+n$ is even. In (4) $\mathrm{K}_{0}(x)$ is the modified Bessel function, $r_{1}$ and $r_{2}$ are the appropriate particle co-ordinates in the transverse plane and $\psi_{i}$ are normalised transverse wavefunctions. All other symbols have their usual meanings.

The zero temperature two-subband model with only the 
ground state occupied implies that $\Pi_{22}(q, \omega)$ vanishes identically, and the plasmon dispersion relation reduces to [3]

$$
\begin{aligned}
& {\left[1-V_{1111}(q) \Pi_{11}(q, \omega)\right]} \\
& \times\left[1-V_{1212}(q)\left\{\Pi_{12}(q, \omega)+\Pi_{21}(q, \omega)\right\}\right]=0
\end{aligned}
$$

The intrasubband plasmon is obtained from

$$
1-V_{1111}(q) \Pi_{11}(q, \omega)=0
$$

and the intersubband excitation from

$$
1-V_{1212}(q)\left\{\Pi_{12}(q, \omega)+\Pi_{21}(q, \omega)\right\}=0
$$

In equations (5) to (7) the real parts of the polarizability are used, with the imaginary parts defining the single particle continua. Further we have employed the symmetry condition

$$
V_{1212}(q)=V_{2112}(q)=V_{1221}(q)=V_{2121}(q)
$$

It is evident that within this theoretical model the effects of different cross-sectional geometries will be manifest in the form factors (4) (hence Coulomb matrix elements), and, as mentioned earlier, the subband energy separation $\Delta E$.
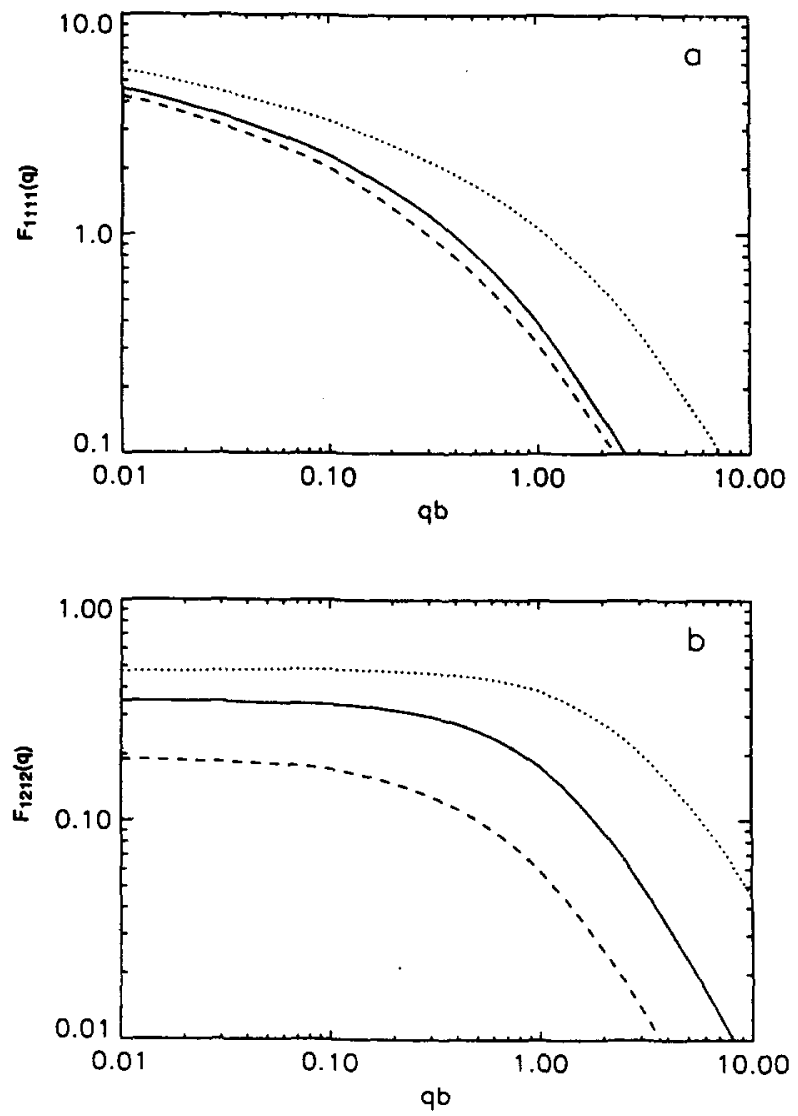

Figure 1. The structure factors for (a) $F_{1111}(q)$ and (b) $F_{1212}(q)$ against $q b$ where the solid curve corresponds to the circle, the dotted curve the 2:1 ellipse and the dashed curve the 10:1 ellipse.
Figure 1 compares the structure factors for a circle of radius $b$, an ellipse with semi-major axis, $a$, twice the semiminor axis, $b,(2: 1$ ellipse) and a 10:1 ellipse. It is seen that for $F_{1111}(q)$ the value for the circle is not too different from that of the 10:1 ellipse. This surprising result is due to the behaviour of the ground state wavefunction as the eccentricity of the ellipse increases. For a large eccentricity, it has been demonstrated elsewhere [17] that the electronic wavefunction is confined to the regions of lowest curvature, i.e. away from the 'corners'. This is in contrast to the behaviour in the rectangular case, where the wavefunctions have a sinusoidal form whatever the dimensions of the rectangle. Despite such manifest differences in wavefunction characteristics, the difference between the three curves in figure 1 is not too great for small values of $q b$ (the regime relevant to the numerical determination of the plasmon modes).

The structure factor, $F_{1212}(q)$, appropriate for the determination of the intersubband plasmon, is seen from figure 1 to be almost constant in the long wavelength region. Here the difference between the three curves is rather more pronounced. This, together with the differences in $\Delta E$, are responsible for the geometrical dependence of the intersubband plasmon energy.

For comparison, we depict in figure 2 the analogous structure factors for a wire with rectangular cross-section of width $2 a$ and breadth $2 b$, for $a=b$ and $a=2 b$ (2:1 rectangle)
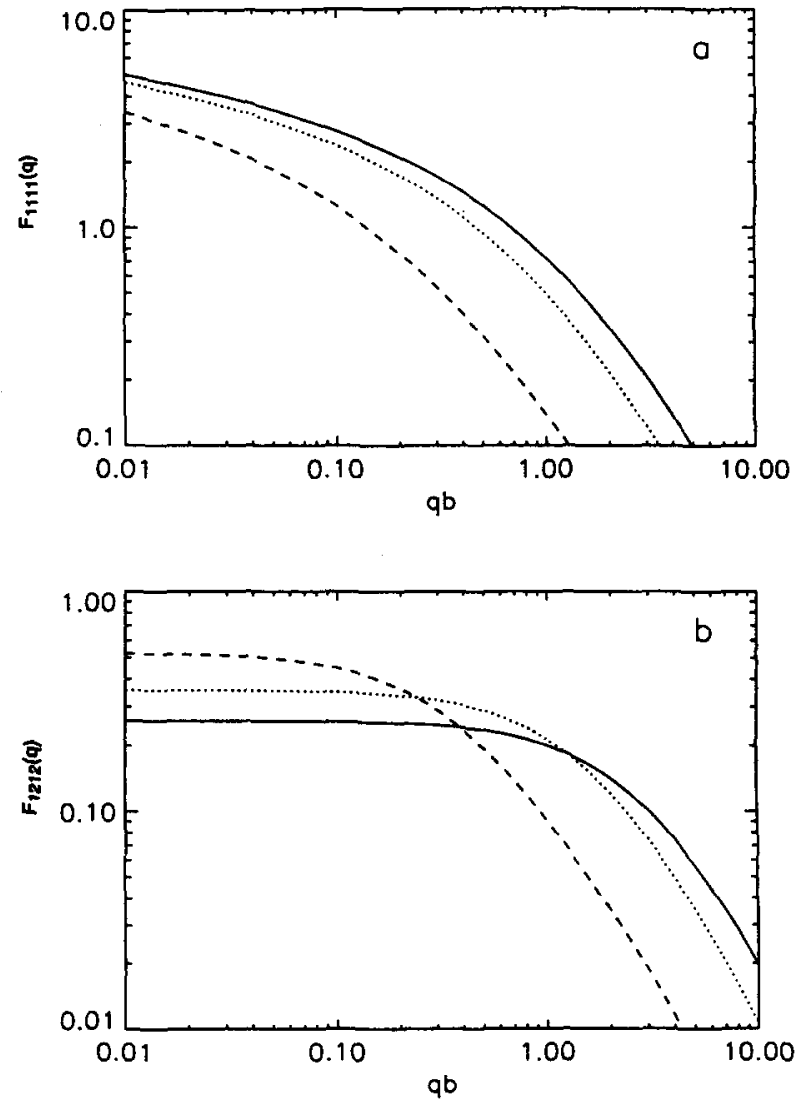

Figure 2. The structure factors for (a) $F_{1111}(q)$ and (b) $F_{1212}(q)$ against $q b$ where the solid curve corresponds to the square, the dotted curve the $2: 1$ rectangle and the dashed curve the 10:1 rectangle. 

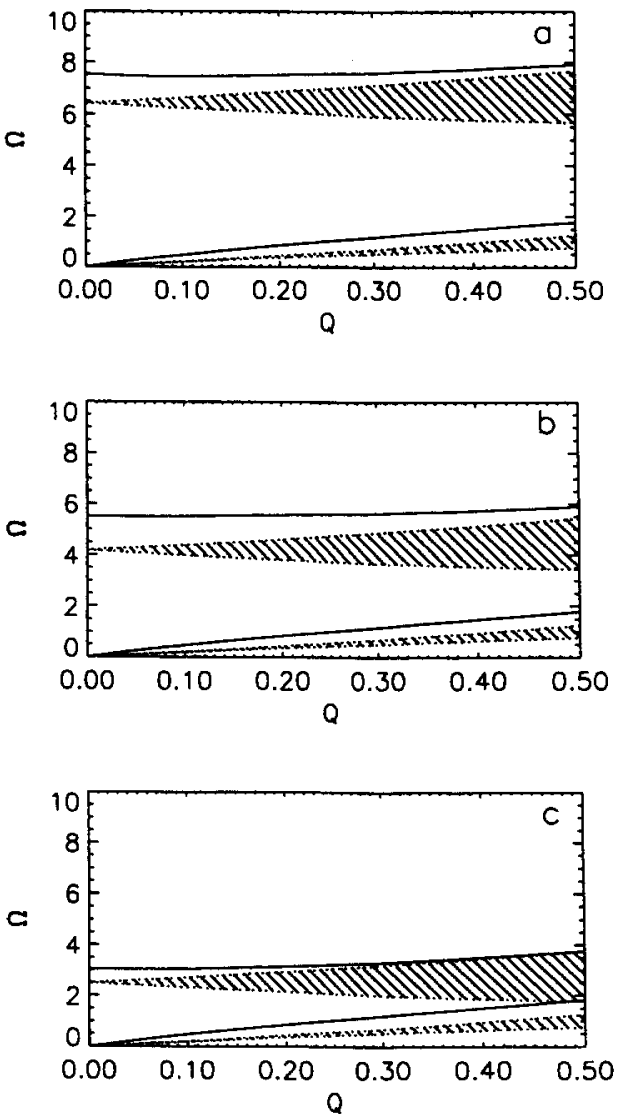

Figure 3. The plasmon spectra for (a) the circle (b) the 2:1 ellipse and (c) the 10:1 ellipse. The shaded regions correspond to the single particle continua.

and a 10:1 rectangle. It is seen that the order of the curves is different from the elliptical case for $F_{1111}(q)$ (figure 2a), in particular the square and the 2:1 rectangle give comparable values. Again, for small $a b$, the different curves give comparable values, to each other and to the corresponding elliptical case. Figure $2 \mathrm{~b}$ illustrates $F_{1212}(q)$ for the rectangular wire. For small $q b$, the order of the curves is reversed compared to figure $2 a$. In particular, there is a marked difference between the long wavelength value of $F_{1212}(q)$ for the 10:1 rectangular wire compared to that of the 10:1 ellipse. This is explained by the different behaviour of the wavefunctions in the two cases [17].

We now come to the numerical evaluation of the plasmon dispersion relation. It is convenient to introduce the following dimensionless variables $\Omega=\hbar \omega / E_{f}$ and $Q=q / k_{f}$ together with

$$
\begin{aligned}
& A^{-1}(q)=\frac{m^{*} e^{2}}{\pi^{3} \varepsilon \varepsilon_{o} n} \times \begin{cases}F_{1111}(q) & \text { intra } \\
F_{1212}(q) & \text { inter }\end{cases} \\
& \Omega_{ \pm}=Q^{2} \pm 2 Q+\frac{\Delta E}{E_{F}}
\end{aligned}
$$

( $\Omega_{ \pm}$for the intrasubband plasmon is given by $(10)$ with $\Delta E=0$ ). Note that the single particle continua are defined
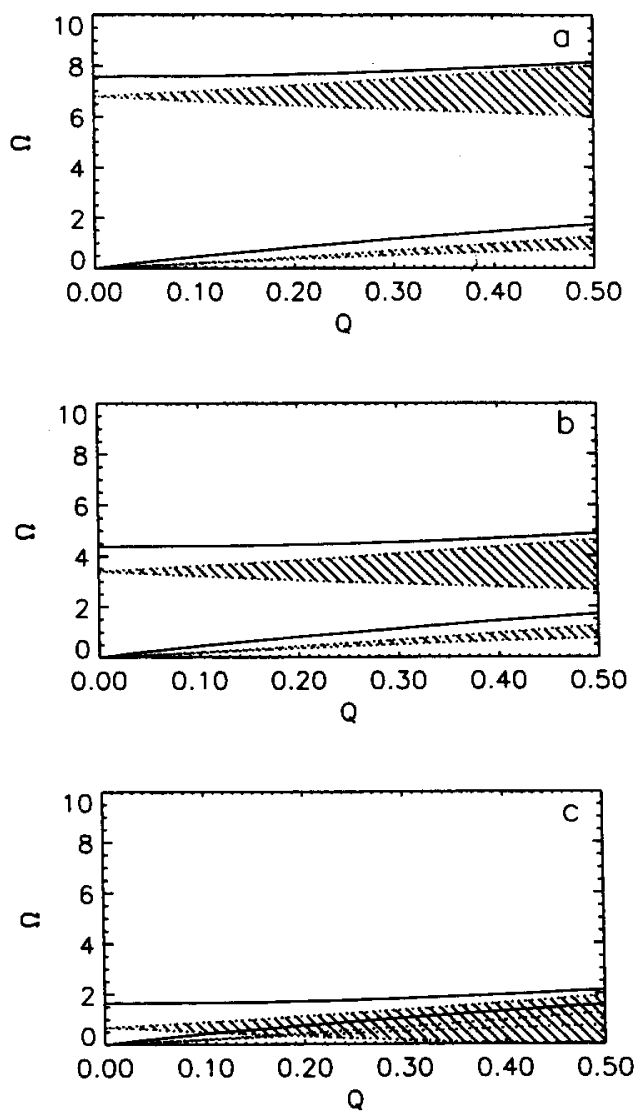

Figure 4. The plasmon spectra for (a) the square (b) the 2:1 rectangle and (c) the 10:1 rectangle. The shaded regions correspond to the single particle continua.

by

$$
\left|\Omega_{-}\right| \leq \Omega \leq \Omega_{+}
$$

with the $Q=0$ value for the intersubband continuum yielding the subband separation. The plasmon energy $\Omega$ is given for both inter- and intra-subband modes by

$$
\Omega^{2}=\frac{e^{A Q} \Omega_{+}^{2}-\Omega_{-}^{2}}{e^{A Q}-1}
$$

It is instructive to consider the long wavelength limit of (12). We obtain for the intrasubband mode

$$
\Omega \approx \sqrt{\frac{8}{A}} Q \approx q \mid \ln \left(\frac{q x}{2}\right)^{\frac{1}{2}}
$$

with $x$ a characteristic length dependent on the crosssectional geometry. This long wavelength expansion is well established [e.g.7]. A similar expansion for the intersubband excitation yields the following result in agreement with [3]

$$
\Omega^{2}=\left(\frac{\Delta E}{E_{f}}\right)^{2}+\frac{8 \Delta E}{A(q \rightarrow 0) E_{f}}
$$


The second term on the right hand side is the depolarisation shift which depends on the cross-sectional geometry via $\Delta E$ and, as it turns out, to a lesser extent on $A(q)$.

Figure 3 shows the dispersion relation for the plasmons corresponding to the wires of a circle of radius $150 \AA$, a $2: 1$ ellipse and a 10:1 ellipse both with the same area as the circle. It is seen that as the eccentricity of the ellipse increases, the energy of the intrasubband plasmon is hardly changed. In contrast, the intersubband plasmon energy decreases as the eccentricity increases due mainly to the reduction in the subband separation, although the change in the structure factor also contributes. Figure 4 illustrates the plasmon spectrum corresponding to the rectangular wires of figure 2 all with the same area as the circle in figure 3 . The trends displayed in moving from square, 2:1 to 10:1 are similar to those of figure 3 . It is more revealing to compare corresponding curves between figures 3 and 4 . A comparison of figures $3 \mathrm{a}$ and $4 \mathrm{a}$ (circle and square) shows that both the intra- and inter- subband plasmons are practically the same for both geometries. Comparisons of figures $3 b$ and $4 b$, and $3 c$ and $4 c$ on the other hand, illustrate quite clearly differences in the intersubband plasmon frequencies of around $3.5 \mathrm{meV}$, which is within experimental resolution [8-10]. Further, the intersubband single particle regions are also significantly different. It is therefore of interest to investigate experimentally such geometric effects.

\section{CONCLUSIONS.}

In this communication, we have considered the effects of cross-sectional geometry on the plasmon modes of quantum wires. We kept within the two subband RPA regime for simplicity, although extension to higher subbands is not too difficult, the algebra being rather more involved. We have considered circular, elliptical and rectangular wires, all having the same cross-sectional areas and linear carrier concentration. Numerical calculations illustrated differences in the Coulomb matrix elements between the different geometries, and in the resultant intersubband plasmons. It was also shown that the intrasubband plasmon is practically unaffected by any change in geometry, and this is expected to hold for the rather more realistic cross-sections studied recently [15]. The intersubband plasmon is shown to be more sensitive to the wire geometry, and it should therefore be of interest to investigate these excitations for other, more complex geometries.

It is worth noting that the assumption of an infinite barrier, which has been employed here and elsewhere [e.g. 2,7] need not be the only one considered. In fact, harmonic confining potentials have also been used [e.g.5], and an investigation along the lines carried out in this communication using harmonic potentials would also be of interest, especially because harmonic potentials do not produce depolarisation shifts (to first order) as a consequence of the generalised Kohn's theorem [5]. Work along these lines is now in progress.

Acknowledgements: CRB would like to thank Bilkent University for their kind hospitality, the Bloomfield Memorial Trust for a travel award, and EPSRC for a studentship. BT thanks the University of Essex for their kind hospitality and TUBITAK for partial support. NCC would like to thank the EPSRC for financial support.

\section{REFERENCES}

1. W.I. Friesen and B. Bergersen, J. Phys.C: Solid St. Phys.: 136627 (1980).

2. A. Gold and A. Ghazali, Phys. Rev. B41 7626 (1990).

3. Q.P. Li and S. Das Sarma, Phys. Rev. B43 11768 (1991); B44 6277 (1991).

4. L. Wendler et al, Phys. Rev. B43 14669 (1991).

5. R. Haupt $e t$ al, Phys. Rev. B44 13635 (1991).

6. B. Tanatar and N.C. Constantinou, Phys. Rev. B48 18280 (1993).

7. L. Wendler and V.G. Grigoryan, Phys. Stat. Sol. (b) 181133 (1994).

8. A.R. Goni et al, Phys. Rev. Lett. 673298 (1991).

9. R. Strenz et al, Semicond. Sci. Technol. 9399 (1994).

10. For a review see D. Heitmann in, Physics of Nanostructures, eds. J.H. Davies and A.R. Long, (IOP Publishing, Bristol, 1992) page 229.
11. Ben Yu-Kuang Hu and S. Das Sarma, Phys. Rev. Lett. 681750 (1992): Phys. Rev. B48 5469 (1993).

12. R. Tonucci et al, Science 258783 (1992).

13. E. Kapon et al, Phys. Rev. Lett. 63430 (1989).

14. S. Tsukamoto et al, Appl. Phys. Lett. 6249 (1993).

15. C. J. Anthony and M.J. Kelly, Semicond. Sci. Technol. 935 (1994).

16. N.W. McLachlan, Theory and Applications of Mathieu Functions, (Clarendon, Oxford, 1947).

17. C.R. Bennett et al, submitted to J. Phys.: Condens. Matter. 